\title{
Acoustic Analysis of a Mechanical Circulatory Support
}

Laila Hubbert, Per Sundbom, Matthias Loebe, Bengt Peterzén, Hans Granfeldt and Henrik

Casimir Ahn

\section{Linköping University Post Print}

\section{Tweet}

N.B.: When citing this work, cite the original article.

Original Publication:

Laila Hubbert, Per Sundbom, Matthias Loebe, Bengt Peterzén, Hans Granfeldt and Henrik Casimir Ahn, Acoustic Analysis of a Mechanical Circulatory Support, 2014, Artificial Organs, (38), 7, 593-598.

http://dx.doi.org/10.1111/aor.12244

Copyright: (C) 2013 The Authors. Artificial Organs published by Wiley Periodicals, Inc. on behalf of International Center for Artificial Organ and Transplantation (ICAOT).

This is an open access article under the terms of the Creative Commons AttributionNonCommercial-NoDerivs License

http://eu.wiley.com/WileyCDA/

Postprint available at: Linköping University Electronic Press

http://urn.kb.se/resolve?urn=urn:nbn:se:liu:diva-112071 
in treatment of chronic heart failure). Circulation 2004;110: 975-81.

3. Nosé Y, Yoshikawa M, Murabayashi S, Takano T. Development of rotary blood pump technology: past, present, and future. Artif Organs 2000;24:412-20.

4. AlOmari AH, Savkin AV, Stevens M, et al. Developments in control systems for rotary left ventricular assist devices for heart failure patients: a review. Physiol Meas 2013;34:R1-27.

5. Salamonsen RF, Lim E, Gaddum N, et al. Theoretical foundations of a Starling-like controller for rotary blood pumps. Artif Organs 2012;36:787-96.

6. Utkin VI. Scope of the theory of sliding modes. In: Dickinson BW, Fettweis A, Massey JL, Modestino JW, Sontag ED, Thoma M, eds. Sliding Modes in Control and Optimization. Berlin: Springer-Verlag, 1992;116.

7. Hall AW, Soykan O, Harken AH. Physiologic control of cardiac assist devices. Artif Organs 2008;20:271-5.

8. Bullister E, Reich S, Sluetz J. Physiologic control algorithms for rotary blood pumps using pressure sensor input. Artif Organs 2002;26:931-8.

9. Goodwin GC, Graebe SF, Salgado ME. Design via optimal control techniques. In: Goodwin et al., eds. Control System Design. Upper Saddle River, NJ: Prentice Hall, 2001;240.

10. Guyton AC, Hall JE. Textbook of Medical Physiology. Philadelphia, PA: Elsevier Saunders, 2006.

11. AlOmari AH, Javed F, Savkin AV, et al. Non-invasive measurements based model predictive control of pulsatile flow in an implantable rotary blood pump for heart failure patients. Conference Proceeding IEEE Mediterranean of Control and Automation. 2011;491-6.

12. Arndt A, Nüsser P, Graichen K, Müller J, Lampe B. Physiological control of a rotary blood pump with selectable therapeutic options: control of pulsatility gradient. Artif Organs 2008;32:761-71.

13. Edwards C, Spurgeon S. Sliding mode design approaches. In: Reilly OJ, Rogers E, ed. Sliding Mode Control: Theory and Applications. Boca Raton, FL: CRC Press, 1998;7.

14. Gao W, Wang Y, Homaifa A. Discrete-time variable structure control systems. IEEE Trans Ind Electron 1995;42:11722.

15. Spurgeon SK. Hyperplane design techniques for discrete-time variable structure control systems. Int J Control 1992;55:44556.

16. Lim E, Dokos S, Cloherty SL, et al. Parameter-optimized model of cardiovascular-rotary blood pump interactions. IEEE Trans Biomed Eng 2010;57:254-66.

17. Wu Y, Paul A, Tao G, Wood H, Olsen D, Tribble C. An advanced physiological controller design for a left ventricular assist device to prevent left ventricular collapse. Artif Organs 2003;27:926-30.

18. Lim E, AlOmari AH, Savkin AV, et al. A method for control of an implantable rotary blood pump for heart failure patients using non-invasive measurements. Artif Organs 2011;35:E17480.

19. Choi S, Antaki JE, Boston R, Thomas D. A sensorless approach to control of a turbodynamic left ventricular assist system. IEEE Trans Control Syst Technol 2001;3:473-82.

20. Choi S, Boston JR, Antaki JF. Hemodynamic controller for left ventricular assist device based on pulsatility ratio. Artif Organs 2007;31:114-25.

21. McIntyre KM, Sasahara AA. The hemodynamic response to pulmonary embolism in patients without prior cardiopulmonary disease. Am J Cardiol 1971;28:288-94.

22. Slaughter MS, Bartoli CR, Sobieski MA, et al. Intraoperative evaluation of the Heartmate II flow estimator. J Heart Lung Transplant 2009;28:39-43.

23. Petersen IR, Ugrinovskii VA, Savkin AV. Robust Control Design Using H-infinity Methods. London: Springer-Verlag, 2000.

24. Savkin AV, Evans RJ. Hybrid Dynamical Systems. Controller and Sensor Switching Problems. Boston, MA: Birkhauser, 2002.
(C) 2013 The Authors. Artificial Organs published by Wiley Periodicals, Inc. on behalf of International Center for Artificial Organ and Transplantation (ICAOT).

This is an open access article under the terms of the Creative Commons Attribution-NonCommercial-NoDerivs License, which permits use and distribution in any medium, provided the original work is properly cited, the use is non-commercial and no modifications or adaptations are made.

\section{Acoustic Analysis of a Mechanical Circulatory Support}

\author{
* *Laila Hubbert, * *Per Sundbom, \$Matthias Loebe, \\ †Bengt Peterzén, †Hans Granfeldt, \\ and $\$$ Henrik Ahn \\ *Clinical Department of Cardiology at the Heart \\ Center, University Hospital of Linkoping; \\ tDepartment of Cardiovascular Surgery and \\ Anesthesia at the Heart Center, University Hospital \\ of Linkoping; $¥$ Department of Medicine \& Health, \\ Linkoping University, Linkoping, Sweden; \\ and \$Division of Transplant and Assist Devices at \\ Methodist DeBakey Heart \& Vascular Center, \\ Houston, TX, USA
}

Abstract: Mechanical circulatory support technology is continually improving. However, adverse complications do occur with devastating consequences, for example, pump thrombosis that may develop in several parts of the pump system. The aim of this study was to design an experimental clot/thrombosis model to register and analyze acoustic signals from the left ventricular assist device (LVAD) HeartMate II (HMII) (Thoratec Corporation, Inc., Pleasanton, CA, USA) and detect changes in sound signals correlating to clots in the inflow, outflow, and pump housing. Using modern telecom techniques, it was possible to register and analyze the HMII pump-specific acoustic fingerprint in an experimental model of LVAD support using a mock loop. Increase in pump speed significantly $(P<0.005)$ changed the acoustic fingerprint at certain frequency $(0-23000 \mathrm{~Hz})$ intervals (regions: R1-3 and peaks: P1,3-4). When the ball valves connected to the tubing were narrowed sequentially by $\sim 50 \%$ of the inner diameter (to mimic clot in the out- and inflow tubing), the frequency spectrum changed significantly $(P<0.005)$ in $\mathrm{P} 1$ and $\mathrm{P} 2$ and $\mathrm{R} 1$ when the outflow tubing was narrowed. This change was not seen to the same extent when the lumen of the ball valve connected to the inflow tube was narrowed by $\sim 50 \%$. More significant $(P<0.005)$ acoustic changes were detected in $\mathrm{P} 1$ and $\mathrm{P} 2$ and $\mathrm{R} 1$ and R3, with the largest dB figs. in the lower frequency ranges in R1 and P2, when artificial clots and blood clots passed through the pump system. At higher frequencies, a significant change in $\mathrm{dB}$ figs. in $\mathrm{R} 3$ and $\mathrm{P} 4$ was detected when clots passed through the pump system. Acoustic monitoring

\section{doi:10.1111/aor.12244}

Received July 2013; revised September 2013.

Corresponding Author: Dr. Laila Hubbert, Department of Cardiology at the Heart Center, University Hospital-Departments of Medicine \& Health, Linkoping University, Linkoping 581 85, Sweden. E-mail: Laila.Hubbert@lio.se 
of pump sounds may become a valuable tool in LVAD surveillance. Key Words: Mechanical circulatory supportFrequency analysis-Thrombosis.

The worldwide use of mechanical circulatory support (MCS) has grown rapidly (1). Outcome continues to improve, but complications such as embolic events related to treatment with MCS are feared $(2,3)$. Pump thrombosis formation can occur even if the patient apparently has adequate anticoagulation. The development of a pump thrombosis is difficult to detect, and an embolic event may be the first symptom suffered by the patient. Our clinical finding of an altering sound from the left ventricular assist device (LVAD) HeartMate II (Thoratec Corporation, Inc., Pleasanton, CA, USA) (HMII), heard through a regular stethoscope in recipients before the occurrence of a thromboembolic event, has resulted in the ongoing SoundMate study, where patients with an HMII perform daily acoustic recordings at home and transmit them to the study center via a smartphone (4). A continuous flow MCS generates a typical sound that is easily registered by routine auscultation. A temporary or permanent change in sound would then indicate a change in pump function. The cause of this phenomenon is not clear. One suggestion is that a sudden change in sound may reflect an imminent pump thrombosis located within the pump and/or in the in- and/or outflow cannula.

Slaughter et al. in 2007 published results from a study on nine patients receiving the pulsatile assist device HM XVE where acoustic signals from the assist device were collected and analyzed using a hydrophone data acquisition system, including a sensor, an AD converter, and a data storage system, which was correlated to the electrocardiogram. They found that acoustic signal monitoring successfully identified HM XVE device end of life (5).

Makino et al. have published data from animal studies on an automatic diagnosis system designed for detecting early stages of malfunction of an artificial heart. The automatic diagnosis system was based on an electronic stethoscope system. Using this system, it was possible to identify initial signs of malfunction of the artificial heart (6). Bowles et al. presented two cases at the International Society for Heart and Lung Transplantation (ISHLT) meeting 2011, where acoustic changes showing typical frequency spectrum peaks were observed in association with pump thrombosis in the HeartWare centrifugal blood pump (7). At the ISHLT annual meeting in 2013, a group from Sydney showed in an experimental model as well as in patients that by analyzing the peaks in the frequency spectrum, pump thrombosis could be detected (8).
To our knowledge, there is no article published, apart from abstracts, on acoustic data from continuous flow devices $(4,9)$.

The aim of this study was to design an experimental in vitro model to register and analyze acoustic signals from the HMII continuous flow MCS, and to detect changes in sound correlating to artificial and blood clot/thrombosis, using modern telecom techniques.

\section{MATERIALS AND METHODS}

\section{Experimental setting}

The HMII pump was placed in a plastic bag filled with water to allow sound recordings via a smartphone. To mimic the clinical situation of sound recording, the smartphone's microphone was attached to the plastic bag at approximately $3 \mathrm{~cm}$ from the pump housing.

Measurements to detect acoustic changes with increases in speed recorded with HMII pumping at speeds of 6000-10 $000 \mathrm{rpm}$ were performed with the pump in a closed circuit and performed earlier. For that reason, these recordings were not used as baseline. The experimental setting was performed in eight steps at $8000 \mathrm{rpm}$, with the HMII in an open circuit to facilitate rinsing of the pump system between clots. Different artificial thrombosis (Clot) situations were arranged: pump out- and inflow tube narrowed by a ball valve (one ball valve at a time was introduced to the circuit); a gelatin clot of two different viscosities (5 and $10 \mathrm{~g}$ gelatin of animal origin, Oetker [Bielefeld, Germany], in $0.2 \mathrm{~L}$ water each and prepared $12 \mathrm{~h}$ in advance); and finally, a human blood clot passing through the pump. The pump was flushed with water for 10 min between clots, and the experiments were repeated three times for each situation. For each clot, the sounds were recorded and the HMII monitor power consumption (watts) and flow (L/min) were registered.

1 Baseline 1: recorded with the ball valve connected to the inflow tubing and before narrowing of the tube.

2 Pump inflow tube narrowed 50\%: the inflow tube was narrowed by $50 \%$ of the inner diameter. The ball valve was then removed.

3 Baseline 2: recorded with the ball valve connected to the outflow tubing and before narrowing of the tube.

4 Pump outflow tube narrowed 50\%: the outflow tube was narrowed by $50 \%$ of the inner diameter. The ball valve was then removed.

5 Baseline 3: open tubes with no ball valves connected. 
6 Clot 1 through the pump: $20 \mathrm{~mL}$ of a gelatin formulation with low viscosity was injected into the inflow tube.

7 Clot 2 through the pump: $20 \mathrm{~mL}$ gelatin formulation with higher viscosity was injected into the inflow tube.

8 Human blood clot through the pump: a blood clot collected after removal of thoracic drainage from a patient that had undergone cardiac surgery the day before was finally injected $(20 \mathrm{~mL})$ into the inflow tubing.

\section{Acoustic recordings}

Sounds from the HMII in the different experimental settings named previously were recorded using an iPhone (Apple, Inc., Cupertino, CA, USA) with the commercially available stethoscope application iStethPro (Dr. Peter J Bentley, London, UK) and transferred via telecommunication to a frequency analysis software program (Audacity 1.3.13-beta, Unicode, Ash, Chinen and Crook, Pittsburgh, PA, USA) and analyzed. Three 8-s sound samples from each experiment were collected and analyzed.

\section{Frequency spectrum and acoustic analysis}

Sound is composed of multiple sound waves of different frequencies (Hertz: fluctuations or unit/ time) and amplitudes (sound strength). It is possible to analyze the noise level at each frequency and also present the data as a frequency spectrum graph with amplitude on the $y$-axis in decibel $(\mathrm{dB})$ and frequency on the $\mathrm{x}$-axis in Hertz $(\mathrm{Hz})$. The frequency measurements are set by the software program between 0 and $23000 \mathrm{~Hz}$, and at 255 different standardized frequency levels with $400 \mathrm{~Hz}$ intervals. In this analysis, the description of amplitude is as noise level $(-\mathrm{dB})$.

The acoustic fingerprint from an HMII has the broadest frequency spectrum between 1000 and $10000 \mathrm{~Hz}$. In this interval, a peak is seen at $\sim 1000 \mathrm{~Hz}$, and an additional smaller peak at $\sim 7000 \mathrm{~Hz}$ may be present. At higher frequencies, peaks around $\sim 15000$ and $\sim 22000 \mathrm{~Hz}$ are commonly present. For that reason, the frequency spectrum in the present study was divided into different frequency regions (R1-3) and peaks (P1-4) in order to facilitate calculations and clarify changes in frequencies of the spectrum during each experimental situation (R1: 1000$6500 \mathrm{~Hz}$; R2: 8500-14 $000 \mathrm{~Hz}$; R3: 15 000-21 $000 \mathrm{~Hz}$; P1: 0-1000 Hz; P2: 6500-8500 Hz; P3: 14 00015000 Hz; P4: 21 000-23 000 Hz) (Fig. 1).

All data are presented as mean and standard deviation. Changes were calculated using the twotailed Student's $t$-test and a $P$ level of $<0.05$ was considered significant.

\section{RESULTS}

It was possible to collect and analyze acoustic data from HMII in the experimental setting using available telecom techniques, and the baseline acoustic fingerprint of this specific HMII was registered.

\section{Change in pump speed}

Changes with increases in pump speed are shown in Table 1. The overall increases in amplitude due to increase in speed are similar to those seen in a previous study (9). With each increase in pump speed, significant changes were detected in regions R1-R3 and in $\mathrm{P} 1, \mathrm{P} 3$, and $\mathrm{P} 4$, but not $\mathrm{P} 2$. $\mathrm{P} 2$ is not sensitive to increases in pump speed (Table 1).

\section{Changes seen with the different clot situations}

As the introduction of a ball valve to a tube may change the flow conditions, a baseline measurement was performed between the different clot experiment settings. There were minor nonsignificant changes in baseline measurements between the open tube and that when the open ball-valve was attached to the out- or inflow tubing.

When the ball valves, connected one at a time, were sequentially narrowed by $50 \%$ of the inner diameter, the frequency spectrum changed significantly $(P<0.005)$ in $\mathrm{P} 1-2$ and $\mathrm{R} 1$ when the outflow tubing was narrowed. This change was not seen to the same extent when the ball valve was connected to the inflow tube followed by narrowing the lumen by $\sim 50 \%$. When artificial clots and human blood clots passed through the pump system, similar but more distinct acoustic changes were detected in $\mathrm{P} 1$ and $\mathrm{P} 2$ and $\mathrm{R} 1$ and R3, most occurring in the lower frequencies in R1 and P2 (Fig. 1). At higher frequencies, significant changes in $\mathrm{dB}$ in $\mathrm{R} 3$ and $\mathrm{P} 4$ were detected when clots passed through the pump system (Table 1).

\section{Pump monitor}

At the three baseline measurements, the power was 4.0-3.9-4.2 $\mathrm{W}$ and the flow was 3.8-3.8-3.9 L/min. After narrowing the in- and outflow tubing, the power decreased and the flow was reduced compared with the original output (pump inflow tube narrowed $50 \%: 3.2 \mathrm{~W}$ and $1.9 \mathrm{~L} / \mathrm{min}$, and pump outflow tube narrowed $50 \%: 3.2 \mathrm{~W}$ and $2.1 \mathrm{~L} / \mathrm{min}$ ). When the artificial clots passed through the pump, there was a decrease in power (Clot.1: 2.7 W and Clot.2: 2.4 W), but when the blood clots passed through the pump, there was a $48 \%$ increase in power to $7.4 \mathrm{~W}$. No flow data were presented on the monitor when the three different clots passed through the pump. 

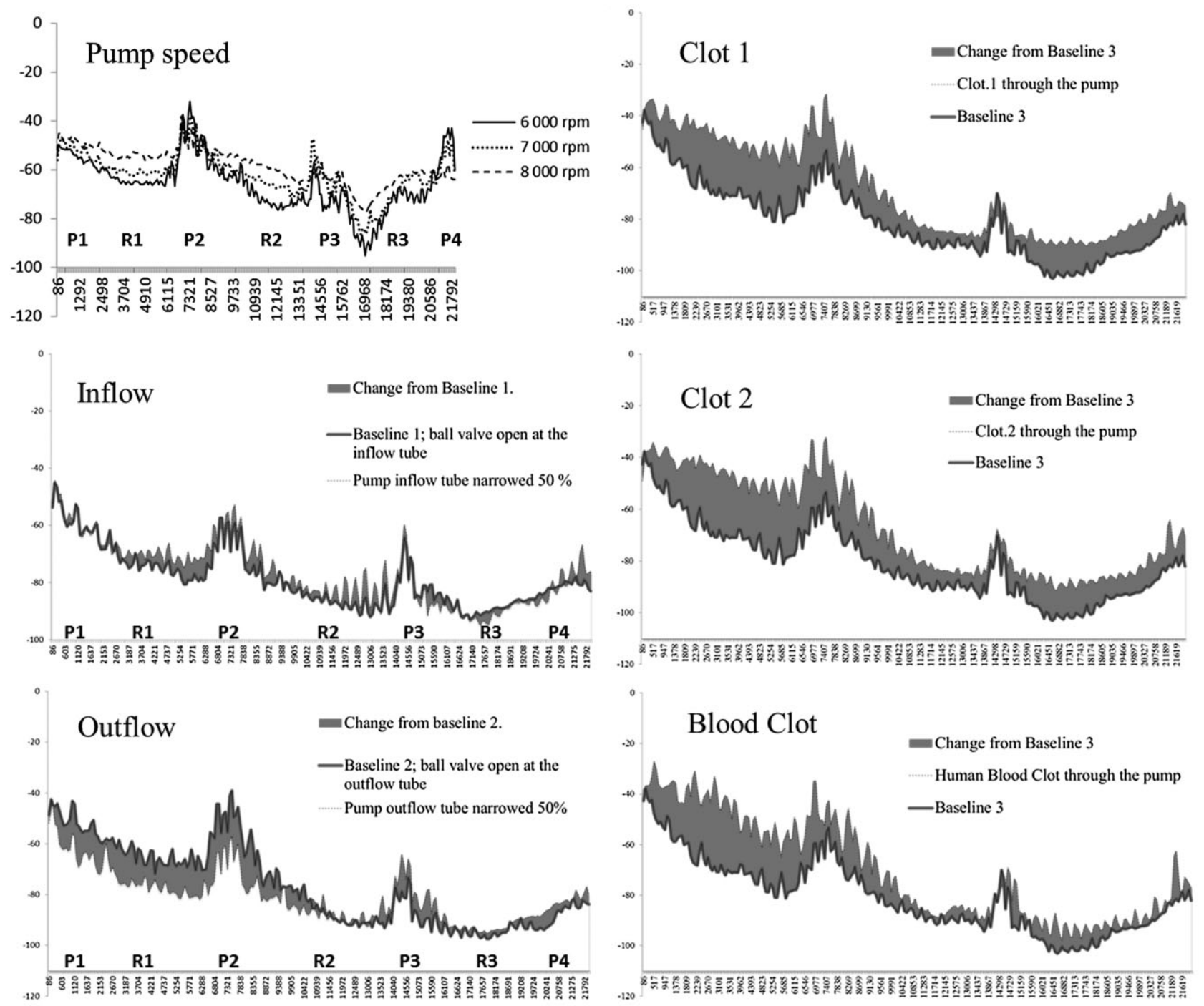

FIG 1. Change in the frequency spectrum at increased pump speed curves (upper left panel) shows example of change in acoustic fingerprint when pump speed increased from 6000 to 7000 to $8000 \mathrm{rpm}$.

Acoustic changes in gray, when narrowing the inflow and outflow tubes with $50 \%$, respectively (left panel), and at different clots: two different viscosities of clots and a human blood clot (right panel) passed through the pump. $R=$ regions (R1: 1000-65 000; R2: 8500-14 000; R3: 15 000-21 000) Hz. P = peaks (P1: 0-1000; P2: 6500-8500; P3: 14 000-15 000 P4; $21000-23$ 000) Hz. Frequency $(x$-axis) in $0-23000 \mathrm{~Hz}$, and amplitude ( $y$-axis) in $-\mathrm{dB}$.

\section{DISCUSSION}

LVAD has become an important treatment option for patients with advanced heart failure, and technology is continually improving (2,10-12). However, adverse complications do occur and may have devastating consequences (3). Pump thrombosis may develop at various locations in the pump system, resulting in thromboembolic events, changes in pre- and afterload, or pump failure. In this experimental model, we used artificial and human blood clots in an attempt to mimic the sound properties of a pump clot/thrombosis. With the present experimental model, using commercially available telecom techniques, we found it possible to register sounds from the HMII and to define a baseline acoustic fingerprint and its variation with increases in pump speed. One frequency interval, P2 was found to be insensitive to changes in speed.

Each HMII has its own acoustic fingerprint in the experimental setting as well as in the clinical situation. Any change from the baseline acoustic pattern (fingerprint), at a given pump speed, appears to be of importance. It is difficult to compare the actual $\mathrm{dB}$ figures at different speeds as the $\mathrm{dB}$ scale is logarithmic and will only be comparable in the same region or peak, and should be valued as a change from the 
baseline. The peaks at $\sim 1000, \sim 7000, \sim 15000$, and $\sim 22000 \mathrm{~Hz}$, which are commonly present in the acoustic fingerprint of the HMII, may depend on the characteristics of sound waves in liquid, and the size and configuration of the pump, or the record devices, and have no known clinical significance.

Water was used in this experimental model in order to maintain a stable level of viscosity during the different parts of the experiment, and to avoid interaction between heparinized blood and the clots. The acoustic properties of sounds transmitted through the chest wall, together with the noise from blood flow in the heart and major vessels in the clinical situation, and those transmitted through water in a plastic bag as in the experimental model, are not comparable. This study did not show if a change in the acoustic fingerprint signifies turbulence, a vortex phenomenon, large eddies from narrowing of the in- and/or outflow, or a normal change in the pump's natural frequency range. Further animal studies on the interaction between the heart, hemodynamics, and blood clotting in acoustic analyses are needed if we are to confirm that experimental measurements can be extrapolated to a clinical system for the detection of pump thrombosis.

The same recording devices and analyzing techniques used in the ongoing SoundMate study were chosen for this study as it is patient friendly, easily usable, and a currently available technique. Further technical models will reveal if this is the optimal technique for this purpose. The lower parts of the frequency spectrum, that showed the greatest changes when clots passed through the pump, are also the frequencies chosen to be prominent in the sounddetecting devices (optimized for voice and music). Whether another system, including software, recording device, microphones, or accelerometers, either without or with other low- and high-pass filters, will reveal other regions and peaks of interest is yet to be determined.

This study shows similar significant changes in the specific acoustic fingerprint regardless of whether it was an artificial or a human blood clot that passed through the pump system, but different changes when the out- or inflow tubes were narrowed by $50 \%$. This suggests that the spectral changes observed contain information about where the clot/thrombosis is located in the pump system, and might be dependent on the direct influence of the clot material on the impeller in the pump house, compared with the change in flow, resistance, and pressure when narrowing the tubes.

In the clinical setting, a sudden increase in power consumption at a fixed pump speed has aroused suspicion of pump thrombosis (3). The sensitivity of this phenomenon is not known due to diagnostic 
difficulties with current available diagnostic methods for detecting pump thrombosis, including echocardiography, CT scanning, blood samples, or echocardiographic ramp test (13). In this study, we used an acute clot model, but in the clinical situation the most common scenario is probably that the clot/ thrombosis builds up over a longer period of time and includes fibrin as well as fresh clot material in its structure. Due to the difference in clots as well as the hemodynamics between this experimental situation and clinical situation, our power analyses are not directly applicable clinically. It is well known from clinical experience that the first symptoms suffered by a patient with pump thrombosis are sometimes neurological events, including stroke, despite no change in power consumption of the pump. This study shows that even when the pump is filled with material that causes a change in sound and decreases the flow, it does not necessarily induce a rise in power level, so the acoustic analysis cannot be validated against power at this stage. In future clinical studies, the correlation between acoustic analysis and power consumption will hopefully be revealed.

\section{CONCLUSION}

This study shows similar significant changes in the acoustic fingerprint of a HeartMate II regardless of whether an artificial or a human blood clot passed through the pump system. Our results indicate that acoustic monitoring of pump performance may become a future tool in left ventricular assist device surveillance.

\section{REFERENCES}

1. Kirklin JK, Naftel DC, Kormos RL, et al. Fifth INTERMACS annual report: risk factor analysis from more than 6000 mechanical circulatory support patients. J Heart Lung Transplant 2013;32:141-56.

2. Frazier OH, Rose EA, Oz MC, et al. Multicenter clinical evaluation of the HeartMate vented electric left ventricular assist system in patients awaiting heart transplantation. J Thorac Cardiovasc Surg 2001;122:1186-95.

3. Meyer AL, Kuehn C, Weidemann J, et al. Thrombus formation in a HeartMate II left ventricular assist device. $J$ Thorac Cardiovasc Surg 2008;135:203-4.

4. Hubbert L, Jorde UP, Peterzén B, et al. Early results from the SoundMate Study. Acoustic analysis of a thromboembolic event in a patient treated with HeartMate II (TM), mechanical circulatory support. J Heart Lung Transplant 2012;31:134-5.

5. Slaughter MS, Feldman CM, Sobieski MA, et al. Utilization of acoustic signatures to identify HeartMate XVE device end-oflife. J Heart Lung Transplant 2007;26:579-83.

6. Makino T, Okamoto E, Tanaka S, et al. Estimation of earlystage malfunction using implantable artificial heart sound in animal experiments. Artif Organs 2006;30:360-4.

7. Bowles CT, Noor M, Schmidt A, et al. Successful treatment of implantable rotary left ventricular assist device (LVAD) thrombus is associated with normalisation of acoustic properties. J Heart Lung Transplant 2011;30:S98.
8. Markey P, Dhital K, Gupta S, et al. The value of pump audio signal in patients with left ventricular assist device. J Heart Lung Transplant 2013;32:S30.

9. Hubbert L, Peterzén B, Granfeldt H, Ahn H. Acoustic analysis of a mechanical circulatory support. J Heart Lung Transplant 2012;31:250.

10. Miller LW, Pagani FD, Russell SD, et al. Use of a continuousflow device in patients awaiting heart transplantation. $N$ Engl $J$ Med 2007;357:885-96.

11. Park SJ, Tector A, Piccioni W, et al. Left ventricular assist devices as destination therapy: a new look at survival. J Thorac Cardiovasc Surg 2005;129:9-17.

12. Rose EA, Moskowitz AJ, Packer M, et al. The REMATCH trial: rationale, design, and end points. Randomized Evaluation of Mechanical Assistance for the Treatment of Congestive Heart Failure. Ann Thorac Surg 1999;67:723-30.

13. Uriel N, Morrison KA, Garan AR, et al. Development of a novel echocardiography ramp test for speed optimization and diagnosis of device thrombosis in continuous-flow left ventricular assist devices: the Columbia ramp study. J Am Coll Cardiol 2012;60:1764-75.

\section{Testosterone and Hemoglobin in Hemodialysis Male and Female Patients}

\author{
*Robert Ekart, $†$ Milena Taskovska, $¥$ Nina Hojs, \\ $¥$ Sebastjan Bevc, and $¥$ Radovan Hojs \\ Departments of $*$ Dialysis and $\$$ Nephrology, Clinic \\ for Internal Medicine, University Medical Centre \\ Maribor; and †Medical Faculty, University of \\ Maribor, Maribor, Slovenia
}

\begin{abstract}
It has been speculated that testosterone stimulates erythropoiesis. We hypothesized that hemoglobin levels in hemodialysis (HD) patients are associated with serum testosterone concentrations. Testosterone, hemoglobin, and other biochemical parameters were measured in a representative sample of 98 chronic HD patients $(50$ male, 48 female; age 30-90 years, mean $65 \pm 13.9$ years). We investigated relations among serum testosterone concentration, hemoglobin, ferritin, albumin, body mass index, lean body mass, total cholesterol, low-density lipoprotein and high-density lipoprotein cholesterol, triglycerides, high-sensitivity C-reactive protein (hsCRP), calcium (Ca), $\mathrm{P}$, intact parathyroid hormone, $\mathrm{N}$-terminal pro-brain natriuretic peptide, Karnofsky performance status, and blood pressure (BP) before and after HD. A statistically significant positive correlation between testosterone and hemoglobin was found in all patients $(r=0.25, P<0.01)$, men $(r=0.34, P<0.02)$, but not in women $(r=0.27$, $P=0.07)$. Multiple regression analysis for all patients has shown statistically significant association between hemoglobin and testosterone $(P<0.001)$, hsCRP $(P<0.005)$, lean body mass $(P<0.05)$, post-HD systolic $(P<0.04)$, and
\end{abstract}

\section{doi:10.1111/aor.12218}

Received July 2013, revised September 2013.

Address correspondence and reprint requests to Prof. Robert Ekart, University Medical Centre Maribor, Clinic for Internal Medicine, Department of Dialysis, Ljubljanska 5, Maribor SI-2000, Slovenia. E-mail: robert.ekart2@guest.arnes.si 